\title{
E-Cigarette Promotion on Twitter in Australia: Content Analysis of Tweets
}

Kahlia McCausland ${ }^{1}$, BSc; Bruce Maycock ${ }^{2}, \mathrm{PhD}$; Tama Leaver ${ }^{3}, \mathrm{PhD}$; Katharina Wolf ${ }^{4}, \mathrm{PhD}$; Becky Freeman ${ }^{5}$, $\mathrm{PhD}$; Katie Thomson ${ }^{6}, \mathrm{BSc}$; Jonine Jancey ${ }^{1}, \mathrm{PhD}$

${ }^{1}$ Collaboration for Evidence, Research and Impact in Public Health, School of Public Health, Curtin University, Bentley, Australia

${ }^{2}$ College of Medicine and Health, University of Exeter, Devon, United Kingdom

${ }^{3}$ School of Media, Creative Arts and Social Inquiry, Curtin University, Bentley, Australia

${ }^{4}$ School of Marketing, Curtin University, Bentley, Australia

${ }^{5}$ School of Public Health, University of Sydney, Sydney, Australia

${ }^{6}$ School of Public Health, Curtin University, Bentley, Australia

Corresponding Author:

Kahlia McCausland, BSc

Collaboration for Evidence, Research and Impact in Public Health

School of Public Health

Curtin University

Kent Street

Bentley, 6102

Australia

Phone: 6192667382

Email: kahlia.mccausland@curtin.edu.au

\section{Abstract}

Background: The sale of electronic cigarettes (e-cigarettes) containing nicotine is prohibited in all Australian states and territories; yet, the growing availability and convenience of the internet enable the promotion and exposure of e-cigarettes across countries. Social media's increasing pervasiveness has provided a powerful avenue to market products and influence social norms and risk behaviors. At present, there is no evidence of how e-cigarettes and vaping are promoted on social media in Australia.

Objective: This study aimed to investigate how e-cigarettes are portrayed and promoted on Twitter through a content analysis of vaping-related tweets containing an image posted and retweeted by Australian users and how the portrayal and promotion have emerged and trended over time.

Methods: In total, we analyzed 1303 tweets and accompanying images from 2012, 2014, 2016, and 2018 collected through the Tracking Infrastructure for Social Media Analysis (TrISMA), a contemporary technical and organizational infrastructure for the tracking of public communication by Australian users of social media, via a list of 15 popular e-cigarette-related terms.

Results: Despite Australia's cautious approach toward e-cigarettes and the limited evidence supporting them as an efficacious smoking cessation aid, it is evident that there is a concerted effort by some Twitter users to promote these devices as a health-conducive $(91 / 129,70.5 \%)$, smoking cessation product $(266 / 1303,20.41 \%)$. Further, Twitter is being used in an attempt to circumvent Australian regulation and advocate for a more liberal approach to personal vaporizers (90/1303, 6.90\%). A sizeable proportion of posts was dedicated to selling or promoting vape products (347/1303, 26.63\%), and 19.95\% (260/1303) were found to be business listings. These posts used methods to try and expand their clientele further than immediate followers by touting competitions and giveaways, with those wanting to enter having to perform a sequence of steps such as liking, tagging, and reposting, ultimately exposing the post among the user's network and to others not necessarily interested in vaping.

Conclusions: The borderless nature of social media presents a clear challenge for enforcing Article 13 of the World Health Organization Framework Convention on Tobacco Control, which requires all ratifying nations to implement a ban on tobacco advertising, promotion, and sponsorship. Countering the advertising and promotion of these products is a public health challenge that will require cross-border cooperation with other World Health Organization Framework Convention on Tobacco Control parties. Further research aimed at developing strategies to counter the advertising and promotion of e-cigarettes is therefore needed. 
(JMIR Public Health Surveill 2020;6(4):e15577) doi: 10.2196/15577

\section{KEYWORDS}

electronic cigarette; e-cigarette; electronic nicotine delivery systems; vaping; vape; social media; twitter; content analysis; public health; public policy

\section{Introduction}

In Australia, the context of the present study, the legal status of electronic cigarettes (e-cigarettes) is determined by existing and overlapping laws relating to poisons, therapeutic and consumer goods, and tobacco control [1]. Liquid nicotine is classified as a "Schedule 7-Dangerous Poison" under the Federal Poisons Standard [2], and, as such, the manufacture, sale, or supply of e-cigarettes containing nicotine without lawful authority (ie, prescription from a medical doctor) [3] is prohibited in all Australian states and territories [4]. However, nicotine-containing e-cigarettes can be imported into Australia, as there is no way to determine whether or not an e-cigarette contains nicotine, short of laboratory analysis, which has implications for law enforcement [4,5]. E-cigarettes that do not contain nicotine can be sold in some Australian jurisdictions, provided manufacturers do not make therapeutic claims, while the sale and use of flavored e-liquid are permitted provided it does not contain nicotine [4].

The World Health Organization Framework Convention on Tobacco Control (WHO FCTC) defines tobacco advertising and promotion as "any form of commercial communication, recommendation or action with the aim, effect or likely effect of promoting a tobacco product or tobacco use either directly or indirectly" and requires signatories to the treaty, of which Australia is one, to "undertake a comprehensive ban on all tobacco advertising, promotion and sponsorship" [6]. As nicotine-containing e-cigarettes are banned from retail sale in Australia; the advertising of such products is also not permitted. Further, advertising of all types of e-cigarette products and devices, nonnicotine included, is regulated at the state level, with most states prohibiting any form of advertising or promotion [7-10].

Data from the most recent National Drug Strategy Household Survey [11] report $11.3 \%$ of Australians aged over 14 years have ever used, and $2.5 \%$ currently use, e-cigarettes, increasing from $8.8 \%$ and $1.2 \%$, respectively, since 2016 . These increases occurred in both smokers and nonsmokers and contrast with Australian combustible smoking rates, which have continued to decline over the last 30 years. The most frequent reason for using e-cigarettes reported by people older than 14 years was "out of curiosity" (54.2\%). Further, $22.8 \%$ cited using e-cigarettes because they perceived them to be less harmful than tobacco cigarettes (19.2\% in 2016), and $10.1 \%$ believed vaping to be more socially acceptable than tobacco smoking $(6.0 \%$ in 2016). In addition, $26.9 \%$ of respondents reported they obtained their e-cigarette products online (Australian retailer 12.5\%, overseas retailer $11.1 \%$, unknown origin 3.3\%).

Vaping has become increasingly popular, and awareness, experimentation, and uptake have proliferated both within Australia and globally [12]. Researchers have therefore begun harnessing data from social media to address information gaps, provide timely insights, and inform public policy and public health [13-15]. As of January 2019, there were approximately 2.56 million active monthly Australian Twitter users (64\% male), which equates to approximately $12 \%$ of Australians older than 13 years [16]. Given the popularity of Twitter [16], the high-speed nature of information dissemination, and the significant influence of Twitter as a driver of web traffic [17], insights into how Twitter is used to promote and discuss e-cigarettes are warranted.

Social media's increasing pervasiveness has provided a powerful avenue to market products and influence social norms and behaviors [18]. There is mounting evidence of the volume of e-cigarette promotion on social media [19,20], with studies suggesting adolescents who view e-cigarette social media promotion express greater intention to use e-cigarettes, more positive attitudes toward e-cigarettes, and greater perceptions of e-cigarette use as normative [21,22]. This is concerning, as Australia's current regulatory stance has proven effective in limiting e-cigarette uptake [11]; however, promotion on social media could bring awareness to and encourage experimentation with e-cigarettes or other tobacco products $[23,24]$. The health effects of e-cigarette use are not fully understood; however, a growing body of literature has established acute consequences with even short-term use, with [25] or without nicotine [26,27].

A 2019 scoping review [19] that aimed to identify and describe the messages presented in e-cigarette-related social media promotions and discussions across the United Kingdom, United States, New Zealand, Canada, and Australia identified no studies from Australia. At the time of this study, there was no published literature on how e-cigarettes are promoted and discussed online in the Australian context. We, therefore, aimed to investigate how e-cigarettes are portrayed and promoted on Twitter through a content analysis of related tweets posted and retweeted by Australian users and how the portrayal and promotion have trended over time in the Australian context where e-cigarettes are largely prohibited.

\section{Methods}

\section{Data Collection}

Twitter data were collected via Tracking Infrastructure for Social Media Analysis (TrISMA) [28], a contemporary technical and organizational infrastructure for the tracking of public communication by Australian users of social media. Central to the TrISMA Twitter infrastructure is the Australian Twitter Collection, which continuously gathers tweets from identified Australian accounts (ie, accounts set to an Australian location, geolocation, or time zone or accounts with a description field referring to an Australian location or containing Australia-specific terms) and stores them in a database available to accredited TrISMA researchers. The TrISMA Twitter 
Collection is hosted on a cloud-based Google BigQuery database and accessed through the data visualization tool Tableau.

A list of popular e-cigarette-related terms was developed based on peer-reviewed literature [29-34], trending Twitter hashtags, and frequently co-occurring hashtags (ie, hashtags that appeared in the same caption as the root term), which resulted in 15 keywords: cloudchasing, ecig (includes ecigarette/s), e-cig (includes e-cigarette/s), electroniccig (includes electroniccigarette/s), electronic cigarette (includes electronic cigarettes), eliquid, e-liquid, e-juice, vape (includes vaper and vapes), vaping, vapecommunity, vapefam, vapelife, vapenation, and vapeporn. E-cigarette product names were omitted from the search strategy so as not to bias the results to specific brands [35]. A preliminary search revealed there was minimal Twitter activity using these keywords before 2012 . Therefore, 2 yearly sampling intervals starting from 2012 to 2018 were chosen to maximize the period of time covered while still being able to see the emergence and decline of trends in the collected data.

Data (tweets), along with meta-data information (ie, username, user follower count), were collected from public Australian Twitter users when a tweet included at least one of the identified keywords from either respective year. Data were downloaded in the form of comma-separated values files for each keyword and respective year. Social media users tend to include multiple hashtags within their posts, which resulted in duplicate tweets being collected. Duplicate tweets within keyword corpora for each year and across keyword corpora from the co-use of hashtags were removed, resulting in the inclusion of only unique tweets [36].

Data were assigned a number in ascending order, and 100 tweets from each keyword corpus for each year were randomly selected using an online random sequence generator [37]. Selected data were checked by one researcher (KM) to determine eligibility (ie, written in English and relevant to e-cigarettes). If any of the originally selected 100 tweets did not fit the inclusion criteria, further sampling occurred until 100 eligible tweets were reached. If a keyword corpus had less than 100 tweets, then all eligible tweets were selected. Each tweet was inspected, and, if found to contain an image, a screenshot of the whole post (text and image) was saved for further analysis. Eligible images needed to be stationary (ie, not a video, animated graphic interchange format $[\mathrm{GIF}]$, or other moving content). Only posts that contained an image were included in this study as the influence of the "picture superiority effect," which specifies pictures and images are more likely to be remembered than words, is widely acknowledged [38]. Social media content that includes associated imagery is also more noticeable, shareable, and engaging to users [39].

Retweets (tweets reposted by users) were included in this study, which facilitated the understanding of what information was being circulated by Australian users, even if it originated in another country.

\section{Ethical Considerations}

A particularly salient concern among researchers is whether social media data should be considered public or private data
[40]. Twitter is a social networking service in which users broadcast their opinions and commonly use a hashtag to associate their thoughts on a subject with users on the same subject; therefore, this data is generally referred to as "public data" [40]. For ethical, privacy, and technical reasons, TrISMA does not collect tweets from private accounts or direct messages; therefore, all data collected in this study were publicly available. This study was approved by the Curtin University Human Research Ethics Committee (approval number: HRE2017-0144).

\section{Developing the Coding Framework}

A concept-driven approach (inductive) [41] informed by extant studies [29-34] was utilized to develop a coding framework. The coding frame was tested on a random sample of 100 tweets by 2 researchers (KM and KT), whereby each tweet was read and assigned codes based upon the concepts presented in the descriptive text, hashtags, and accompanying image [42]. It is critical to consider the visual and textual aspects of posts together in the analysis [42] as the study of images can be used to complement and extend the study of health behaviors and may be more valuable than the study of words alone [15]. The 2 researchers followed a hybrid inductive/deductive content analysis approach [41] to refine and further develop the coding framework before transferring the modified framework into IBM SPSS Statistics (v22).

\section{Interrater Reliability Testing}

The 2 researchers applied the modified coding framework to a sample of 140 randomly selected posts (approximately 10\% of the final sample), and an interrater reliability test was performed. Interrater reliability was determined using Krippendorff alpha, and an average score of $\alpha=.89$ was obtained, with a range of .65-1.0, indicating good to perfect agreement [43]. Any discrepancies were discussed to reach consensus, and the coding framework was revised accordingly.

\section{Coding and Analysis}

The final coding framework (Multimedia Appendix 1) was applied by KT and checked for consistency and validity by KM. The coders met regularly to refine coding rules and discuss questions and emergent themes. Each code within the coding framework was a variable in SPSS that functioned as a standalone item and was evaluated as either 1 for present or 2 for absent. Statistical comparisons (ie, between codes and years) were made using chi-square tests or Fisher exact tests, if applicable. Data were analyzed using IBM SPSS Statistics (v22). Due to the small sample size of the 2012 data, a further sensitivity analysis was performed with statistical comparisons made using chi-square and Fisher exact tests to assess the robustness of the results by removing the observations in 2012 .

\section{Results}

\section{Sample of Posts}

Of the 4437 randomly selected tweets, 1553 contained an image, and an eligible sample of 1303 tweets was retained for analysis (Table 1). 
Table 1. Number of posts selected for analysis.

\begin{tabular}{llll}
\hline Year of post & Random sample of posts $(\mathrm{n}=4437), \mathrm{n}$ & Posts containing an image $(\mathrm{n}=1553), \mathrm{n}$ & Posts eligible for analysis $(\mathrm{n}=1303), \mathrm{n}$ \\
\hline 2012 & 570 & 12 & 12 \\
2014 & 1,196 & 289 & 246 \\
2016 & 1,378 & 658 & 540 \\
2018 & 1,293 & 594 & 505 \\
\hline
\end{tabular}

\section{Sensitivity Analysis}

After performing the sensitivity analysis, all associations, except for one, remained significant when removing the 12 observations from 2012. After the removal of the 2012 data, the "quit smoking" association did not retain its significance $(P=.213)$. The results of the sensitivity analysis indicate that, overall, the results were not substantially influenced by the small number of data in 2012 .

\section{Frequency and Description of Codes}

\section{Overview}

In total, 1303 tweets and accompanying images (collectively referred to as posts) were analyzed: 12 from 2012, 246 from 2014, 540 from 2016, and 505 from 2018.

\section{People}

Of the images that contained a person, 60.0\% (326/543) portrayed a man, and the majority of people appeared to be over the age of 18 years (300/313, 95.8\%; Table 2). The largest proportion of people visible in these images was classified as "everyday people" (283/543, 52.1\%). 
Table 2. Frequency statistics for each year corpus and the total sample within the "people" domain.

\begin{tabular}{|c|c|c|c|c|c|}
\hline Associated codes & $2012(\mathrm{~N}=12), \mathrm{n}(\%)$ & $2014(\mathrm{~N}=246), \mathrm{n}(\%)$ & $2016(\mathrm{~N}=540), \mathrm{n}(\%)$ & $2018(\mathrm{~N}=505), \mathrm{n}(\%)$ & Total $(\mathrm{N}=1303), \mathrm{n}(\%)$ \\
\hline People visible & $4(33.3)$ & $115(46.7)$ & $209(38.7)$ & $215(42.6)$ & $543(41.7)$ \\
\hline \multicolumn{6}{|l|}{ Type of people visible } \\
\hline Everyday person & $2(50.0)^{\mathrm{a}}$ & $65(56.5)^{\mathrm{b}}$ & $120(57.4)^{\mathrm{c}}$ & $96(44.6)^{\mathrm{d}}$ & $283(52.1)^{\mathrm{e}}$ \\
\hline Model & $1(25.0)^{\mathrm{a}}$ & $39(33.9)^{\mathrm{b}}$ & $59(28.2)^{\mathrm{c}}$ & $78(36.3)^{\mathrm{d}}$ & $177(32.6)^{\mathrm{e}}$ \\
\hline Celebrity & $1(25.0)^{\mathrm{a}}$ & $4(3.5)^{b}$ & $9(4.3)^{\mathrm{c}}$ & $15(7.0)^{\mathrm{d}}$ & $29(5.3)^{\mathrm{e}}$ \\
\hline Health professional/academic & $0(0)^{\mathrm{a}}$ & $0(0)^{\mathrm{b}}$ & $3(1.4)^{\mathrm{c}}$ & $12(5.6)^{\mathrm{d}}$ & $15(2.8)^{\mathrm{e}}$ \\
\hline Other & $0(0)^{\mathrm{a}}$ & $7(6.1)^{\mathrm{b}}$ & $11(5.3)^{\mathrm{c}}$ & $4(1.9)^{\mathrm{d}}$ & $22(4.1)^{\mathrm{e}}$ \\
\hline Multiple types & $0(0)^{\mathrm{a}}$ & $0(0)^{\mathrm{b}}$ & $7(3.3)^{\mathrm{c}}$ & $10(4.6)^{\mathrm{d}}$ & $17(3.1)^{\mathrm{e}}$ \\
\hline \multicolumn{6}{|l|}{ Gender of people visible } \\
\hline Female & $1(25.0)^{\mathrm{a}}$ & $39(33.9)^{\mathrm{b}}$ & $44(21.0)^{\mathrm{c}}$ & $39(18.1)^{\mathrm{d}}$ & $123(22.7)^{\mathrm{e}}$ \\
\hline Male & $3(75.0)^{\mathrm{a}}$ & $58(50.4)^{b}$ & $134(64.1)^{\mathrm{c}}$ & $131(60.9)^{\mathrm{d}}$ & $326(60.0)^{\mathrm{e}}$ \\
\hline Both & $0(0)^{\mathrm{a}}$ & $7(6.1)^{b}$ & $15(7.2)^{\mathrm{c}}$ & $23(10.7)^{\mathrm{d}}$ & $45(8.3)^{\mathrm{e}}$ \\
\hline Cannot determine & $0(0)^{\mathrm{a}}$ & $11(9.6)^{b}$ & $16(7.7)^{\mathrm{c}}$ & $22(10.2)^{\mathrm{d}}$ & $49(9.0)^{\mathrm{e}}$ \\
\hline \multicolumn{6}{|l|}{ Age of people visible (years) } \\
\hline$<18$ & $0(0)^{\mathrm{f}}$ & $0(0)^{\mathrm{g}}$ & $3(2.6)^{\mathrm{h}}$ & $4(3.2)^{\mathrm{i}}$ & $7(2.2)^{\mathrm{j}}$ \\
\hline$\geq 18$ & $2(100.0)^{\mathrm{f}}$ & $72(100.0)^{g}$ & $111(95.7)^{\mathrm{h}}$ & $115(93.5)^{\mathrm{i}}$ & $300(95.8)^{\mathrm{j}}$ \\
\hline Mixed & $0(0)^{\mathrm{f}}$ & $0(0)^{\mathrm{g}}$ & $2(1.7)^{\mathrm{h}}$ & $4(3.2)^{\mathrm{i}}$ & $6(1.9)^{\mathrm{j}}$ \\
\hline
\end{tabular}

${ }^{\mathrm{a}} \mathrm{N}=4$.

${ }^{\mathrm{b}} \mathrm{N}=115$.

${ }^{\mathrm{c}} \mathrm{N}=209$.

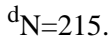

${ }^{\mathrm{e}} \mathrm{N}=543$.

${ }^{\mathrm{f}} \mathrm{N}=2$.

${ }^{\mathrm{g}} \mathrm{N}=72$.

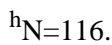

${ }^{\mathrm{i}} \mathrm{N}=123$.

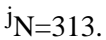

\section{Product Placement and Visibility}

A vaporizer product was visible in $70 \%$ (913/1303) of images, and most commonly $(497 / 1303,38.14 \%)$ these were e-cigarette or other vaping devices (eg, e-hookah, e-cigar; Table 3).
E-cigarette liquids (also known as e-liquid or e-juice) were present in $11.82 \%(154 / 1303)$ of images. In posts that depicted a vaporizer product, the product was placed overtly within the image in $92.7 \%(846 / 913)$ of posts. 
Table 3. Frequency statistics for each year corpus and the total sample within the "vape and tobacco products" domain.

\begin{tabular}{|c|c|c|c|c|c|}
\hline Associated codes & $2012(\mathrm{~N}=12), \mathrm{n}(\%)$ & $2014(\mathrm{~N}=246), \mathrm{n}(\%)$ & $2016(\mathrm{~N}=540), \mathrm{n}(\%)$ & $2018(\mathrm{~N}=505), \mathrm{n}(\%)$ & Total $(\mathrm{N}=1303), \mathrm{n}(\%)$ \\
\hline \multicolumn{6}{|l|}{ Product placement ${ }^{\mathrm{a}}$} \\
\hline Overt & $8(88.9)^{\mathrm{b}}$ & $179(94.2)^{\mathrm{c}}$ & $373(93.5)^{\mathrm{d}}$ & $286(90.8)^{\mathrm{e}}$ & $846(92.7)^{f}$ \\
\hline Covert & $1(11.1)^{\mathrm{b}}$ & $11(5.8)^{\mathrm{c}}$ & $26(6.5)^{d}$ & $29(9.2)^{\mathrm{e}}$ & $67(7.3)^{\mathrm{f}}$ \\
\hline \multicolumn{6}{|l|}{ Product visible } \\
\hline $\begin{array}{l}\text { E-cigarette or another vaping } \\
\text { device }\end{array}$ & $3(25.0)^{b}$ & $116(47.2)^{\mathrm{c}}$ & $199(36.9)^{\mathrm{d}}$ & $149(35.4)^{\mathrm{e}}$ & $497(38.1)^{\mathrm{f}}$ \\
\hline $\begin{array}{l}\text { E-cigarette and another } \\
\text { vape/tobacco product }\end{array}$ & $2(16.7)^{b}$ & $37(15.0)^{\mathrm{c}}$ & $79(14.6)^{\mathrm{d}}$ & $37(7.3)^{\mathrm{e}}$ & $155(11.9)^{\mathrm{f}}$ \\
\hline Vape accessory & $0(0)^{\mathrm{b}}$ & $11(4.5)^{\mathrm{c}}$ & $28(5.2)^{\mathrm{d}}$ & $22(4.4)^{\mathrm{e}}$ & $61(4.7)^{\mathrm{f}}$ \\
\hline $\begin{array}{l}\text { Vape liquid } \\
\text { (e-liquid) }\end{array}$ & $1(8.3)^{\mathrm{b}}$ & $17(6.9)^{\mathrm{c}}$ & $84(15.6)^{\mathrm{d}}$ & $52(10.3)^{\mathrm{e}}$ & $154(11.8)^{\mathrm{f}}$ \\
\hline $\begin{array}{l}\text { Vape liquid and another } \\
\text { vape/tobacco product }\end{array}$ & $1(8.0)^{\mathrm{b}}$ & $0(0)^{\mathrm{c}}$ & $0(0)^{\mathrm{d}}$ & $5(1.0)^{\mathrm{e}}$ & $6(0.5)^{f}$ \\
\hline Showcase in a retail store & $0(0)^{\mathrm{b}}$ & $6(2.4)^{\mathrm{c}}$ & $4(0.7)^{\mathrm{d}}$ & $5(1.0)^{\mathrm{e}}$ & $15(1.2)^{\mathrm{f}}$ \\
\hline Tobacco product & $2(16.7)^{b}$ & $3(1.2)^{\mathrm{c}}$ & $4(0.7)^{\mathrm{d}}$ & $14(2.8)^{\mathrm{e}}$ & $23(1.8)^{\mathrm{f}}$ \\
\hline \multicolumn{6}{|l|}{ Setting } \\
\hline Indoors & $4(66.7)^{\mathrm{g}}$ & $94(77.7)^{\mathrm{h}}$ & $173(71.2)^{\mathrm{i}}$ & $107(60.1)^{\mathrm{j}}$ & $378(69.0)^{\mathrm{k}}$ \\
\hline Outdoors & $2(33.3)^{\mathrm{g}}$ & $27(22.3)^{\mathrm{h}}$ & $70(28.8)^{\mathrm{i}}$ & $71(39.9)^{\mathrm{j}}$ & $170(31.0)^{\mathrm{k}}$ \\
\hline
\end{tabular}

${ }^{\mathrm{a}}$ Only coded for if a product was visible in the post.

${ }^{\mathrm{b}} \mathrm{N}=9$.

${ }^{\mathrm{c}} \mathrm{N}=190$.

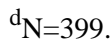

$\mathrm{e}_{\mathrm{N}}=315$.

$\mathrm{f}_{\mathrm{N}=913}$

$\mathrm{g}_{\mathrm{N}}=6$.

$\mathrm{h}_{\mathrm{N}=121 \text {. }}$

${ }^{\mathrm{i}} \mathrm{N}=243$.

${ }^{\mathrm{j}} \mathrm{N}=178$.

$\mathrm{k}_{\mathrm{N}}=548$.

\section{Promotional Practices and Strategies}

In $26.63 \%$ (347/1303) of posts, purchase of e-cigarette products was promoted, and $9.67 \%$ (126/1303) of posts provided Twitter users with a promotional offer (Table 4). Promotional offers could be monetary or nonmonetary, of which nonmonetary offers were most prevalent $(86 / 126,68.3 \%)$. Nonmonetary promotional offers did not lower the cost of a purchase; they instead promoted contests, giveaways, and sweepstakes or offered free shipping or a free gift with purchase. Rather than aiming to sell specific e-cigarette products, some posts promoted vape businesses, brands, and online groups. These posts were categorized as "business listings" and comprised 19.95\% (260/1303) of the total sample (Figure 1). Some business listings and promotional posts used methods to increase their visibility and expand their market, such as operating competitions to win e-cigarette products. However, to enter a competition, Twitter users were required to undertake a series of steps including following the account, and liking, commenting, re-tweeting, or tagging others in the post (Figure 2).

Of posts that displayed or discussed e-liquid products, $71.1 \%$ $(226 / 318)$ described the flavor of the product through either words or images (eg, images of candy or fruits; Figure 3). Creative flavor names (eg, King Cookie Dough, Show me the Honey) and descriptive flavor descriptions (eg, "Grab a sweet and spicy cup of tea from the Chai Wallah as he makes the rounds on an overcrowded train slowly making its way to Varanasi") were commonly depicted in image captions and on product packaging. 
Table 4. Frequency statistics for each year corpus and the total sample within the "promotional practices and strategies" domain.

\begin{tabular}{|c|c|c|c|c|c|}
\hline Associated codes & $2012(\mathrm{~N}=12), \mathrm{n}(\%)$ & $2014(\mathrm{~N}=246), \mathrm{n}(\%)$ & $2016(\mathrm{~N}=540), \mathrm{n}(\%)$ & $2018(\mathrm{~N}=505), \mathrm{n}(\%)$ & Total $(\mathrm{N}=1303), \mathrm{n}(\%)$ \\
\hline E-liquid flavor described (yes) ${ }^{\mathrm{a}}$ & $3(100.0)^{\mathrm{b}}$ & $33(58.9)^{\mathrm{c}}$ & $14490.0)^{\mathrm{d}}$ & $76(76.8)^{e}$ & $226(71.1)^{\mathrm{f}}$ \\
\hline 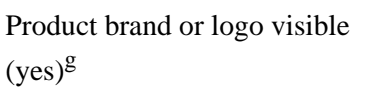 & $4(44.4)^{\mathrm{h}}$ & $83(43.7)^{\mathrm{i}}$ & $230(57.6)^{\mathrm{j}}$ & $144(45.7)^{\mathrm{k}}$ & $461(50.5)^{1}$ \\
\hline $\begin{array}{l}\text { Product brand or logo is visible } \\
\text { anywhere }\end{array}$ & $4(33.3)$ & $128(52.0)$ & $275(50.9)$ & $211(41.8)$ & $618(47.4)$ \\
\hline $\begin{array}{l}\text { Promoting vape product for } \\
\text { purchase }\end{array}$ & $2(16.7)$ & $80(32.5)$ & $164(30.4)$ & $101(20.0)$ & 347 (26.6) \\
\hline Business listing & $2(16.7)$ & $61(24.8)$ & $101(18.7)$ & $96(19.0)$ & $260(20.0)$ \\
\hline Vapor present & $1(8.3)$ & $60(24.4)$ & $104(19.3)$ & $89(17.6)$ & $254(19.5)$ \\
\hline \multicolumn{6}{|l|}{ Promotional offer } \\
\hline Nonmonetary & $1(100.0)^{\mathrm{m}}$ & $14(53.8)^{\mathrm{n}}$ & $29(61.7)^{\mathrm{o}}$ & $45(86.5)^{\mathrm{p}}$ & $89(70.6)^{q}$ \\
\hline Both & $0(0)^{\mathrm{m}}$ & $1(3.8)^{\mathrm{n}}$ & $3(6.4)^{\mathrm{o}}$ & $0(0.0)^{\mathrm{p}}$ & $4(3.2)^{\mathrm{q}}$ \\
\hline Vape product review & $0(0)$ & $7(2.8)$ & $36(6.7)$ & $29(5.7)$ & $72(5.5)$ \\
\hline Cartoon & $1(8.3)$ & $8(3.3)$ & $31(5.7)$ & $18(3.6)$ & $58(4.5)$ \\
\hline Sale notice & $0(0)$ & $3(1.2)$ & $11(2.0)$ & $1(0.2)$ & $15(1.2)$ \\
\hline
\end{tabular}

${ }^{\mathrm{a}}$ Only coded for if the post displayed or discussed an e-liquid product.

${ }^{\mathrm{b}} \mathrm{N}=3$.

${ }^{\mathrm{c}} \mathrm{N}=56$.

${ }^{\mathrm{d}} \mathrm{N}=160$.

${ }^{\mathrm{e}} \mathrm{N}=99$.

${ }^{\mathrm{f}} \mathrm{N}=318$.

${ }^{\mathrm{g}}$ Only coded for if a vaping-related product was visible in the post.

${ }^{\mathrm{h}} \mathrm{N}=9$.

${ }^{\mathrm{i}} \mathrm{N}=190$.

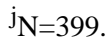

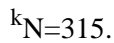

${ }^{1} \mathrm{~N}=913$.

$\mathrm{m}_{\mathrm{N}=1 \text {. }}$.

${ }^{\mathrm{n}} \mathrm{N}=26$.

${ }^{\circ} \mathrm{N}=47$.

$\mathrm{p}_{\mathrm{N}=52 \text {. }}$

${ }^{\mathrm{q}} \mathrm{N}=126$. 
Figure 1. Example within the business listing category of the "promotional practices and strategies" domain.

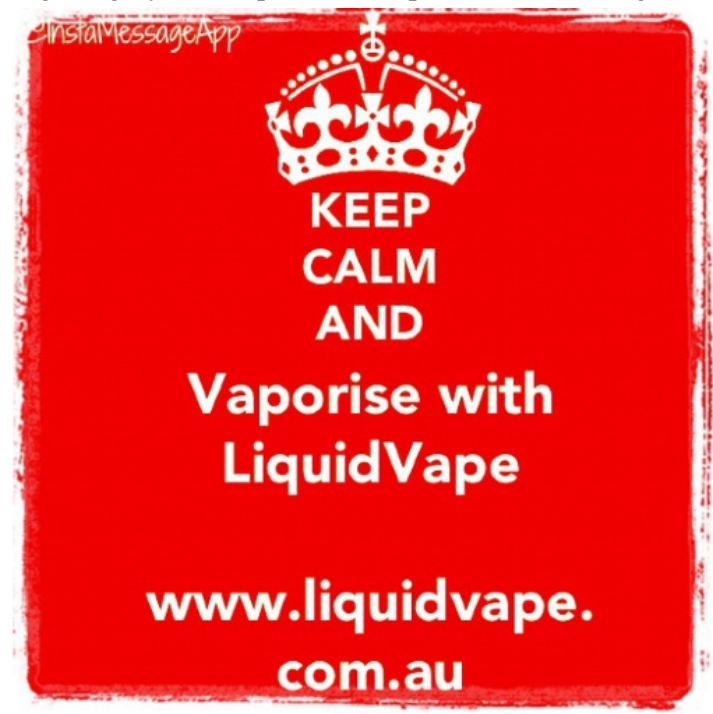

Figure 2. Example within the nonmonetary promotional offer category of the "promotional practices and strategies" domain.

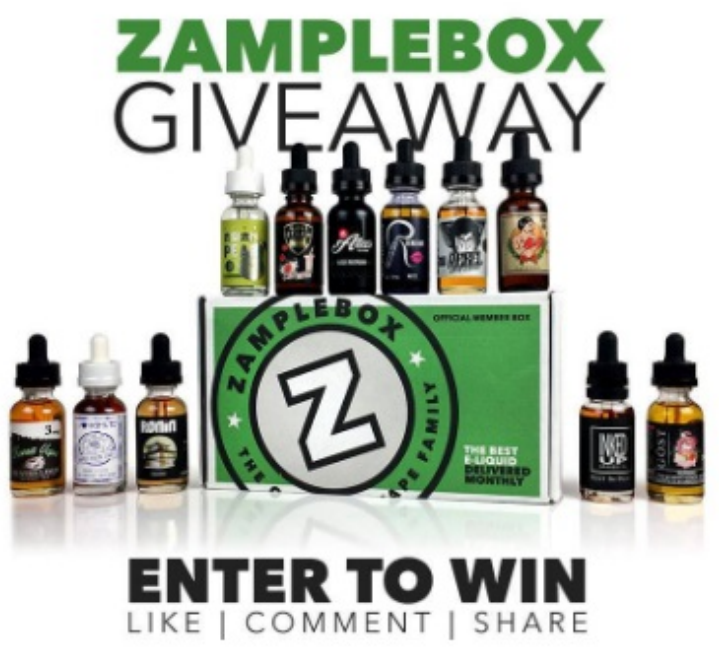

Figure 3. Example of a flavor within the "promotional practices and strategies" domain.

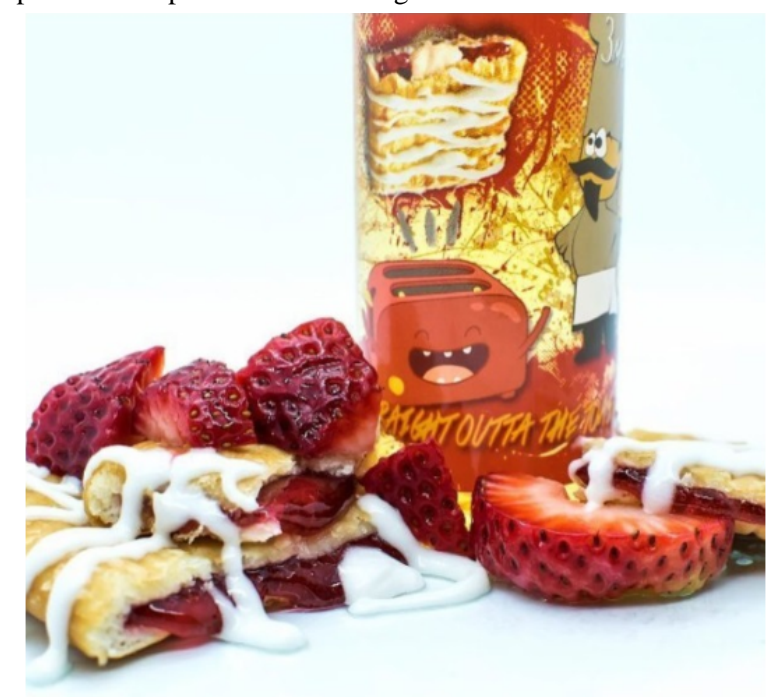




\section{Health, Safety, and Product Claims}

The potential health benefits and consequences (Figure 4) of e-cigarettes were detailed in $9.90 \%$ (129/1303) of posts, of which $70.5 \%$ (91/129) conveyed the perceived benefits associated with e-cigarette use (Table 5). These posts compared e-cigarette products to their presumed more harmful counterpart, combustible cigarettes, by listing the alleged harmless ingredients found in vaporizer products (eg, nicotine, propylene glycol, glycerin, flavoring; Figure 5) compared to the toxic ingredients found in tobacco cigarettes (eg, ammonia, carbon monoxide, lead), labelled e-cigarettes as "smoke-free," publicized that e-cigarettes provide a "safe" or "safer" smoking experience, and included testimonials from people who had quit smoking through the use of e-cigarettes and their subsequent positive changes in health. Further, a significant proportion of posts promoted e-cigarettes as an effective smoking cessation aid (266/1303, 20.41\%; Figure 6).

Figure 4. Example of health consequences being explained within the "health, safety, and product claims" domain.

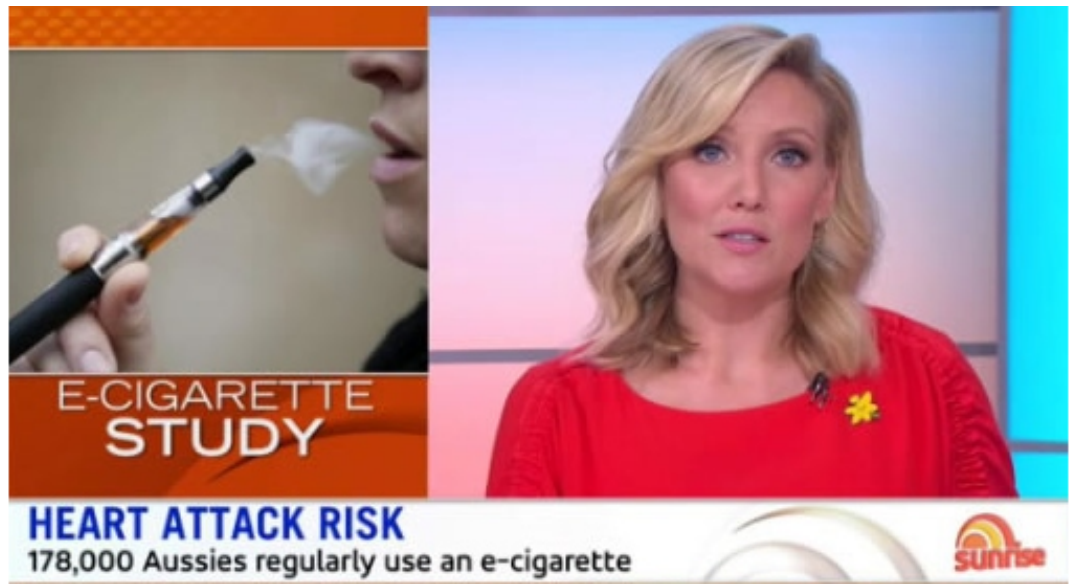

Table 5. Frequency statistics for each year corpus and the total sample within the "health, safety, and product claims" domain.

\begin{tabular}{|c|c|c|c|c|c|}
\hline Associated codes & $2012(\mathrm{~N}=12), \mathrm{n}(\%)$ & $2014(\mathrm{~N}=246), \mathrm{n}(\%)$ & $2016(\mathrm{~N}=540), \mathrm{n}(\%)$ & $2018(\mathrm{~N}=505), \mathrm{n}(\%)$ & Total $(\mathrm{N}=1303), \mathrm{n}(\%)$ \\
\hline Quit smoking & $7(58.3)$ & $52(21.1)$ & $96(17.8)$ & $111(22.0)$ & $266(20.4)$ \\
\hline \multicolumn{6}{|l|}{ Health } \\
\hline Total & $1(8.3)$ & $15(6.1)$ & $34(6.3)$ & $79(15.6)$ & $129(9.9)$ \\
\hline Positive & $1(100.0)$ & $13(86.7)$ & $22(64.7)$ & $55(69.6)$ & $91(75.2)$ \\
\hline Negative & $0(0)$ & $2(13.3)$ & $12(35.3)$ & $24(30.4)$ & $38(29.5)$ \\
\hline Safety & $0(0)$ & $8(3.3)$ & $30(5.6)$ & $24(4.8)$ & $62(4.8)$ \\
\hline Public health & $0(0)$ & $2(0.8)$ & $18(3.3)$ & $30(5.9)$ & $50(3.8)$ \\
\hline Youth vaping & $0(0)$ & $3(1.2)$ & $8(1.5)$ & $31(6.1)$ & $42(3.2)$ \\
\hline $\begin{array}{l}\text { Health warning or age restriction } \\
\text { visible }\end{array}$ & $0(0)$ & $3(1.2)$ & $8(1.5)$ & $14(2.8)$ & $25(1.9)$ \\
\hline \multicolumn{6}{|l|}{ Nicotine $^{a}$} \\
\hline Nicotine level (mg) & $0(0)^{\mathrm{b}}$ & $4(8.7)^{\mathrm{c}}$ & $27(18.1)^{\mathrm{d}}$ & $3(3.9)^{\mathrm{e}}$ & $34(12.4)^{\mathrm{f}}$ \\
\hline Nicotine-free & $1(50.0)^{b}$ & $1(2.3)^{\mathrm{c}}$ & $9(6.0)^{\mathrm{d}}$ & $9(11.7)^{\mathrm{e}}$ & $20(7.3)^{f}$ \\
\hline $\begin{array}{l}\text { Multiple products: nicotine } \\
\text { and nicotine-free }\end{array}$ & $0(0)^{\mathrm{b}}$ & $2(4.3)^{\mathrm{c}}$ & $2(1.3)^{\mathrm{d}}$ & $1(1.3)^{\mathrm{e}}$ & $5(1.8)^{\mathrm{f}}$ \\
\hline No nicotine level visible & $1(50.0)^{\mathrm{b}}$ & $39(84.8)^{\mathrm{c}}$ & $111(74.5)^{\mathrm{d}}$ & $64(83.1)^{\mathrm{e}}$ & $215(78.5)^{f}$ \\
\hline
\end{tabular}

${ }^{\mathrm{a}}$ Only coded for if the post displayed an e-liquid product.

${ }^{\mathrm{b}} \mathrm{N}=2$.

${ }^{\mathrm{c}} \mathrm{N}=46$.

${ }^{\mathrm{d}} \mathrm{N}=149$.

${ }^{\mathrm{e}} \mathrm{N}=77$.

$\mathrm{f}_{\mathrm{N}}=274$. 
Figure 5. Example of an explanation of e-liquid ingredients within the "health, safety, and product claims" domain.

\section{WHAT'S IN E-CIGARETTE LIQUID?}

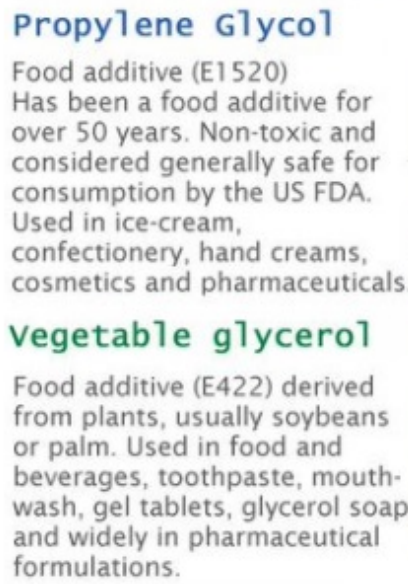

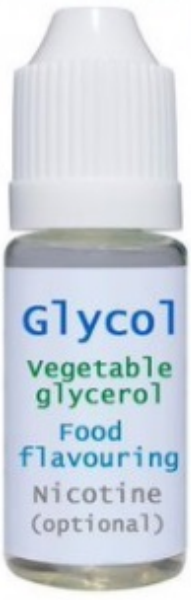

\section{Food flavouring}

E-liquid alone has little taste, so flavour concentrates such as vanilla, mint, fruit, beverage, confectionery, tobacco and spices are usually added.

\section{Nicotine}

Stimulant found in tomatoes, eggplant, cauliflower and especially tobacco leaf.

Typical e-liquid contains a 1 to $3 \%$ concentration of nicotine. A drop of typical e-liquid contains 0.5 to $1 \mathrm{mg}$ of nicotine (compared to nicotine gum with 2 to $4 \mathrm{mg}$ ).

Figure 6. Example of describing e-cigarettes as a smoking cessation aid within the "health, safety, and product claims" domain.

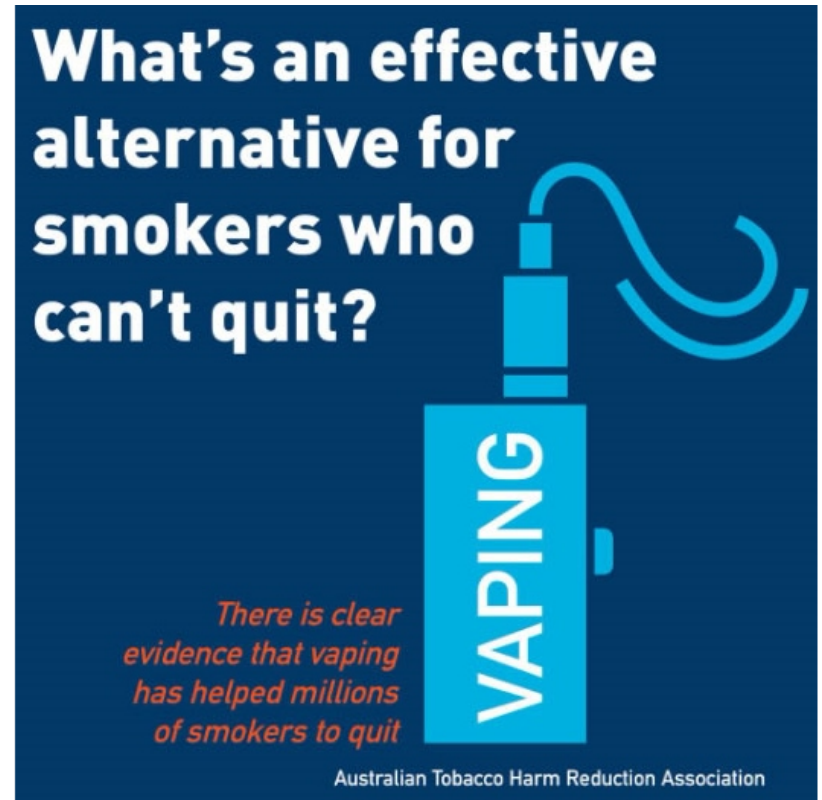

Only $1.92 \%(25 / 1303)$ of posts contained a health warning or age restriction. Health warnings were commonly displayed on e-cigarette product packaging (Figure 7). Age restrictions indicating products were not to be used by those under the age of 18 years were commonly asserted by a small icon, similar to that found on alcoholic beverages in Australia. Of the posts that portrayed an e-liquid product, $21.5 \%$ (59/274) identified whether the product contained nicotine (eg, $2 \mathrm{mg}$ ) or was nicotine-free (eg, $0 \mathrm{mg}$ ). 
Figure 7. Example of a health warning within the "health, safety, and product claims" domain.

\section{WARNING: \\ This product contains nicotine. Nicotine is an addictive chemical.}

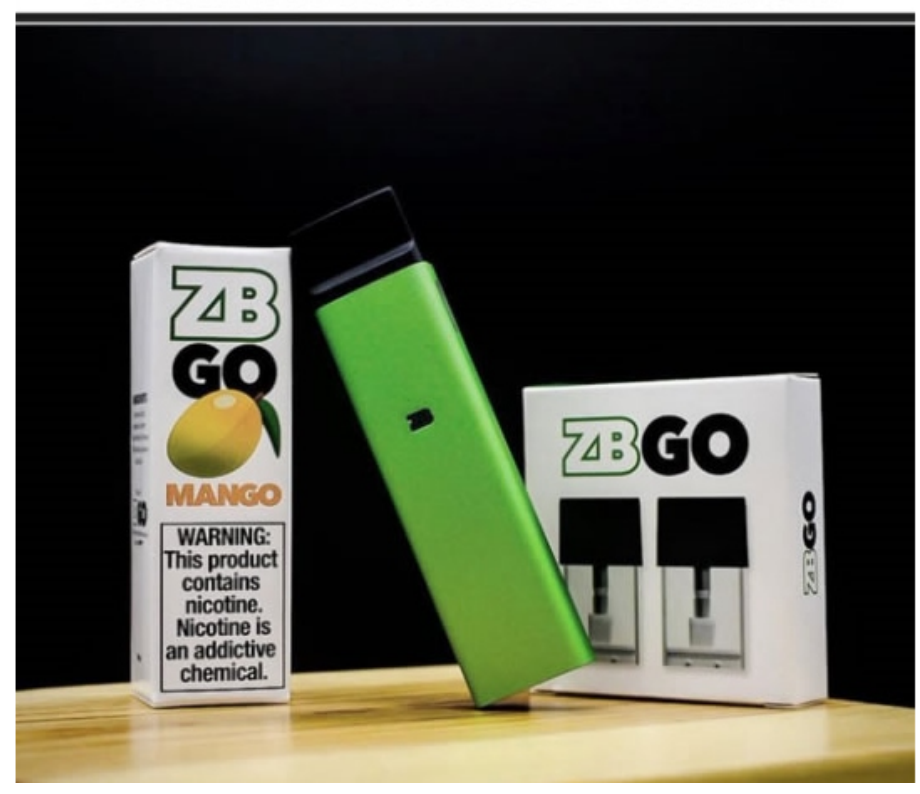

\section{Behaviors and Practices}

Over half $(709 / 1303,54.41 \%)$ of all posts indicated the presence of a vaping community or shared social identity or addiction bond, commonly through the use of hashtags. Popular hashtags that accompanied these posts included \#vapecommunity, \#vapefam, \#vapenation, and \#vapelife. One user posted:

\#vape \#vapefam \#WeVapeWeVote \#vapenation As a show of solidarity, I will add your \#THR [tobacco harm reduction] medal to your profile pic[ture] if you'd like. Simply send me a DM [direct message] $w /$ [with] the picture and it can be done quickly.

"Hand check/product check" posts $(255 / 1303,19.57 \%)$ often appeared as simple photographs of an e-cigarette device or liquid in the hand of its user (Figure 8) or standalone (Table 6). These images were commonly taken in people's homes, cars, and other outdoor locations and were frequently accompanied by the hashtag \#handcheck.

Figure 8. Example of a hand check post within the "behaviors and practices" domain.

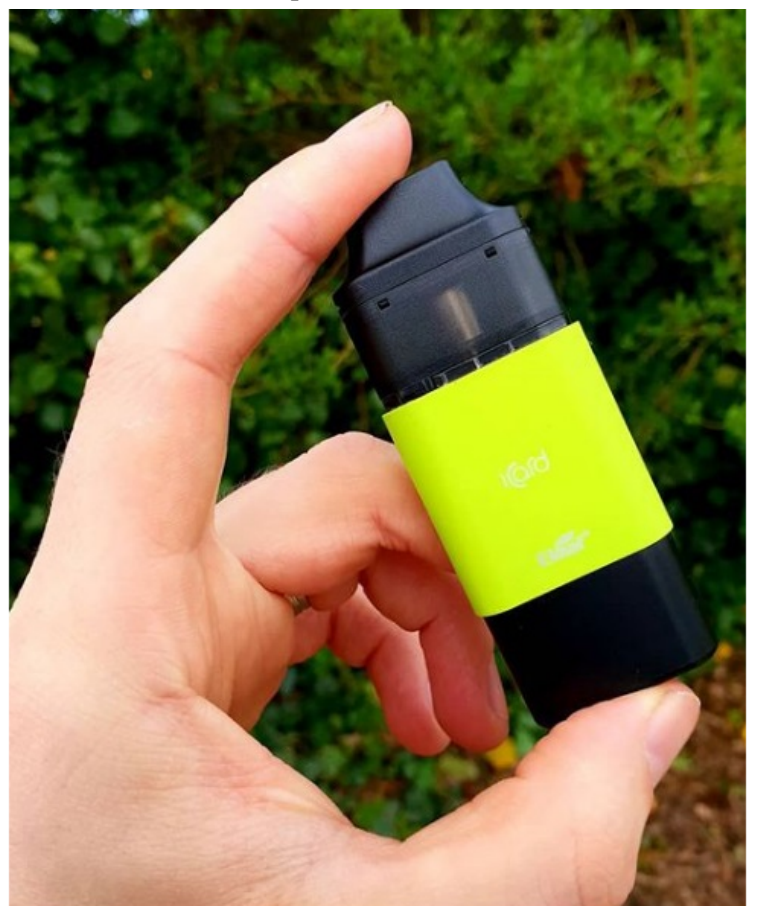


Table 6. Frequency statistics for each year corpus and the total sample within the "behaviors and practices" and "association with another substance" domains.

\begin{tabular}{|c|c|c|c|c|c|}
\hline Associated codes & $2012(\mathrm{~N}=12), \mathrm{n}(\%)$ & $2014(\mathrm{~N}=246), \mathrm{n}(\%)$ & $2016(\mathrm{~N}=540), \mathrm{n}(\%)$ & $2018(\mathrm{~N}=505), \mathrm{n}(\%)$ & Total $(\mathrm{N}=1303), \mathrm{n}(\%)$ \\
\hline \multicolumn{6}{|l|}{ Behaviors and practices domain } \\
\hline Identity or community & $0(0)$ & $142(57.7)$ & $341(63.1)$ & $226(44.8)$ & $709(54.4)$ \\
\hline Hand check/product check & $4(33.3)$ & $45(18.3)$ & $127(23.5)$ & $79(15.6)$ & $255(19.6)$ \\
\hline Selfie & $0(0)$ & $17(6.9)$ & $24(4.4)$ & $14(2.8)$ & $55(4.2)$ \\
\hline Building/DIY $^{\mathrm{a}}$ & $1(8.3)$ & $10(4.1)$ & $21(3.9)$ & $18(3.6)$ & $50(3.8)$ \\
\hline Meme & $0(0)$ & $4(1.6)$ & $17(3.1)$ & $26(5.1)$ & $47(3.6)$ \\
\hline Vape play & $0(0)$ & $12(4.9)$ & $21(3.9)$ & $10(2.0)$ & $43(3.3)$ \\
\hline Person vaping & $1(8.3)$ & $71(28.9)$ & $99(18.3)$ & $90(17.8)$ & $261(20.0)$ \\
\hline Erotic or sexualized & $0(0)$ & $7(2.8)$ & $11(2.0)$ & $1(0.2)$ & $19(1.5)$ \\
\hline \multicolumn{6}{|l|}{$\begin{array}{l}\text { Association with another } \\
\text { substance domain }\end{array}$} \\
\hline Cannabis (including hemp) & $0(0)^{\mathrm{b}}$ & $1(25.0)^{\mathrm{c}}$ & $11(61.1)^{\mathrm{d}}$ & $11(91.7)^{\mathrm{e}}$ & $23(67.6)^{f}$ \\
\hline Alcohol & $0(0)^{\mathrm{b}}$ & $3(75.0)^{\mathrm{c}}$ & $7(38.9)^{\mathrm{d}}$ & $1(8.3)^{\mathrm{e}}$ & $11(32.4)^{\mathrm{f}}$ \\
\hline
\end{tabular}

${ }^{\text {a } D I Y: ~ d o-i t-y o u r s e l f . ~}$

${ }^{\mathrm{b}} \mathrm{N}=0$.

${ }^{\mathrm{c}} \mathrm{N}=4$.

${ }^{\mathrm{d}} \mathrm{N}=18$.

${ }^{\mathrm{e}} \mathrm{N}=12$.

${ }^{\mathrm{f}} \mathrm{N}=34$.

Men were more often represented in selfies $(40 / 55,73 \%$; $P<.001)$, and in posts of people vaping (139/261, 53.3\%; $P<.001)$ and performing vape tricks $(25 / 43,58 \% ; P<.001$; Figure 9) than women (selfies: 12/55, 22\%; vaping: $84 / 261,32.2 \%$; performing vape tricks: $8 / 43,19 \%$ ). Furthermore, men more frequently posted "hand check/product checks" (98/255, 38.4\%; $P<.001)$ and posts that indicated a connection with the vape community or vaper identity $(199 / 709,28.1 \% ; P=.05)$ than women $(12 / 255,4.7 \%$ and $60 / 709,8.5 \%$, respectively). A person was present in 18 of the 19 "erotic or sexualized" posts, of which $16(89 \%)$ images contained women scantily dressed and suggestively posed (Figure 10). The remaining 2 images portrayed a man and woman together.

Figure 9. Example of male representation within the "behaviors and practices" domain.

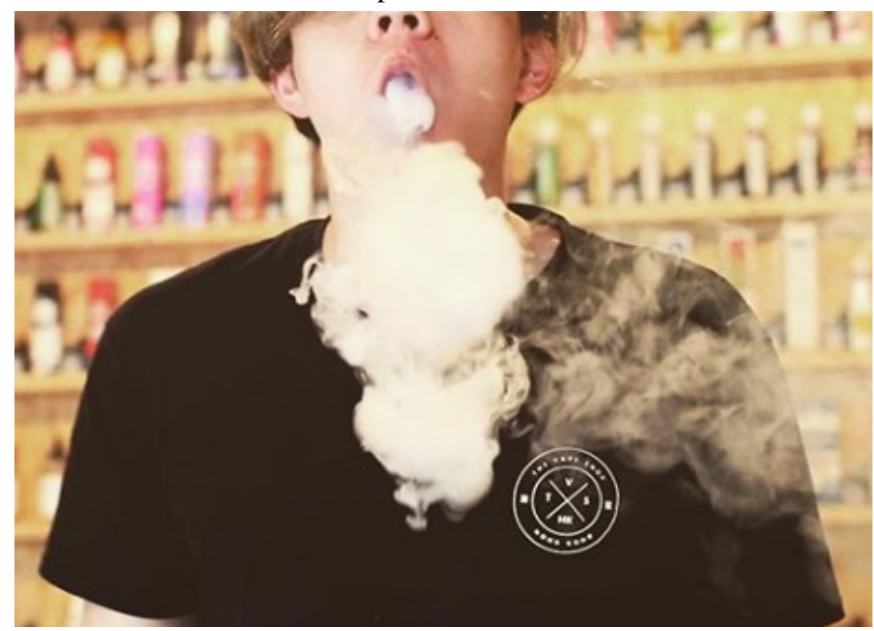


Figure 10. Example of a sexualized image within the "behaviors and practices" domain.

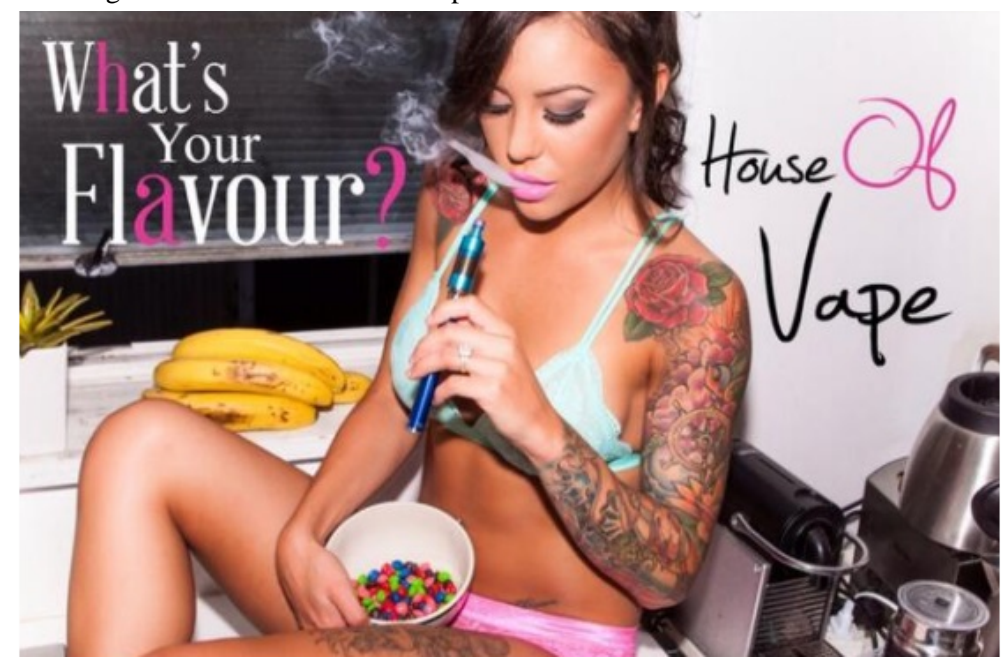

\section{Regulation and Advocacy}

E-cigarette regulation and policy were discussed in $10.74 \%$ (140/1303) of posts (Table 7). An almost equal proportion of posts was found to be discussing or in favor of liberal (90/1303,
$6.91 \%)$ versus restrictive $(87 / 1303,6.68 \%$; Figure 11$)$ e-cigarette policies. Advocacy efforts were encouraged in $4.99 \%$ (65/1303) of posts, of which $60 \%$ (39/65) supported liberal e-cigarette regulation (Figure 12).

Table 7. Frequency statistics for each year corpus and the total sample within the "regulation and advocacy" domain.

\begin{tabular}{llllll}
\hline Associated codes & $2012(\mathrm{n}=12), \mathrm{n}(\%)$ & $2014(\mathrm{n}=246), \mathrm{n}(\%)$ & $2016(\mathrm{n}=540), \mathrm{n}(\%)$ & $2018(\mathrm{n}=505), \mathrm{n}(\%)$ & Total $(\mathrm{n}=1303), \mathrm{n}(\%)$ \\
\hline Regulation or policy & $0(0)$ & $9(3.7)$ & $43(8.0)$ & $100(19.8)$ & $140(10.7)$ \\
Liberal regulation & $0(0)$ & $6(2.4)$ & $26(4.8)$ & $58(11.5)$ & $90(6.9)$ \\
Restrictive regulation & $0(0)$ & $2(0.8)$ & $27(5.0)$ & $58(11.5)$ & $87(6.7)$ \\
Advocacy & $0(0)$ & $3(1.2)$ & $16(3.0)$ & $46(9.1)$ & $65(5.0)$ \\
\hline
\end{tabular}

Figure 11. Example of a restrictive policy within the "regulation and advocacy" domain.

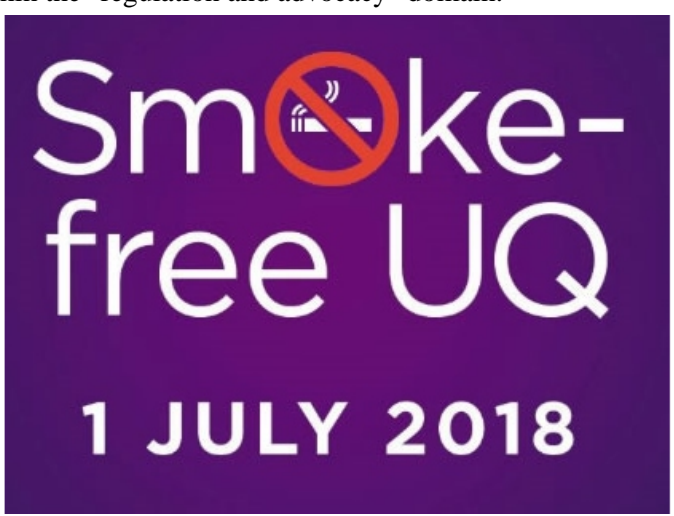


Figure 12. Example of advocacy within the "regulation and advocacy" domain.

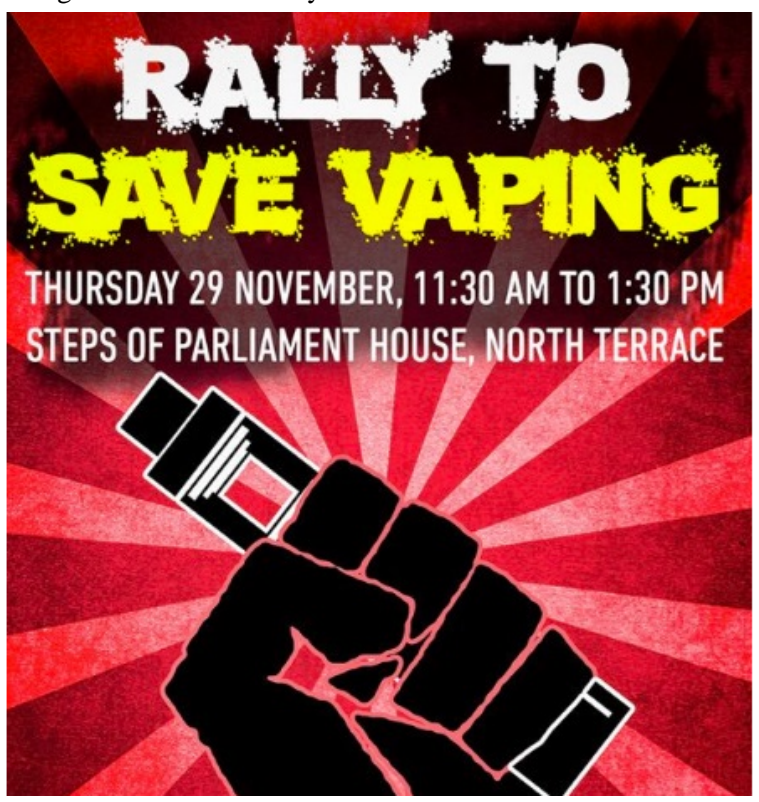

\section{Discussion}

\section{Promotional Practices and Strategies}

The use of several promotional practices and strategies was documented in this study, namely the promotion of positive perceptions of e-cigarette use, implicit and explicit marketing of e-cigarette products and businesses, and the use of promotional offers (monetary and nonmonetary). These findings are consistent with those reported in a recent systematic review of e-cigarette marketing communication [44] and are known and effective strategies utilized by the tobacco industry for decades [45]. These promotional practices coupled with the ease in which consumers can purchase products online through the click of a link have resulted in the exponential growth of online e-cigarette sales worldwide [46]. Investigations into youth online purchasing have confirmed the ease with which young people can purchase e-cigarette products due to the lack of appropriate age detection processes [47-49].

The promotion of e-liquid flavors through images, detailed flavor descriptions, and appealing product packaging was common and is supported by other social media-based investigations [50,51]. E-cigarette users commonly report the importance of flavored e-cigarette products in facilitating smoking abstinence and enhancement of their vaping experience [52]. Subsequently, e-cigarette manufacturers and retailers have adopted the promotion of flavored e-cigarette products as a major marketing strategy [53]. However, evidence indicates the promotion of flavored e-liquid may be particularly attractive to young people [54] and serve as one of the main reasons for e-cigarette initiation [55]. Furthermore, youth have been found to perceive fruit-flavored e-liquids to be less harmful than tobacco-flavored products [56], and fruit-flavored e-liquids have been linked to greater perceived enjoyment [57].

\section{Health, Safety and Product Claims}

It is not uncommon to find posts on social media claiming e-cigarettes are safer than cigarettes and can be used as a cessation tool, with limited or no validation [35]. Only a very small proportion of posts in this study was accompanied by or depicted a health warning or age restriction, and an increasing proportion of posts was found to be promoting the positive health effects of vaping. Furthermore, a substantial proportion of posts promoted e-cigarettes as a replacement or alternative to cigarettes, similar to that found by Laestadius and colleagues [30]. Risk perception plays an important role in product use decision making, and a commonly cited reason for e-cigarette uptake among adults and young people is the belief that they are less harmful than cigarettes [58-60]. Youth who perceive e-cigarettes as harmless or less harmful than cigarettes are at increased susceptibility of uptake compared to youth with more negative views towards vaping $[61,62]$.

\section{Behaviors and Practices}

A common post found in this study, the "hand check/product check," is significant because these posts reflect the variety and wide range of vaporizer and e-liquid products and accessories that exist. As vaporizers continue to evolve, with users able to customize and create unique devices, users are increasingly turning to social media to share the products they are using and creating. Similarly, Chu and colleagues [29] found a large proportion of product-based images posted to the social media platform Instagram exhibiting the hashtag \#handcheck. The authors expressed concern regarding this increasing trend, as these images act as unpaid marketing of e-cigarette products and viewers may interpret these devices to be commonplace and socially acceptable.

The inclusion of hashtags such as \#vapecommunity, \#vapelife, \#vapenation, and \#cloudchaser demonstrate the existence of a vaping identity and community on Twitter, which has also been found in prior vaping-related social media investigations $[30,63]$. Inclusion of such hashtags may function to create an internalization of social bonding and a vape-related identity [63]. This internalization may help one to define who they are and create their own identity and values within a society that has normalized values and practices. This has led to the formation of unique online and face-to-face "vaper" 
communities and identities $[64,65]$, which some people are now adopting and associating with rather than the identity of being a "cigarette smoker" or "ex-smoker." The application of hashtags to social media posts is a form of folksonomy, and the initiating adopters of these electronic tags and subsequent uptake by imitators can be explained by Roger's Diffusion of Innovation Theory, which seeks to explain how, why, and at what rate new ideas and technology spread [66]. It has therefore been suggested by some that these vaping-related discussions may be occurring within some networks as an "echo chamber," whereby the ideas and beliefs of those within the network are strengthened, resulting in the normalization of vaping within these communities [63]. Research examining Australian Twitter users using network analysis methods could provide an Australian perspective on this hypothesis. Further, research that examines how nicotine addiction is represented on social media may assist to understand evolving perceptions of addiction and identity.

\section{Implications for Policy and Research}

This investigation demonstrates that a number of Australian Twitter users are purposefully (commercial) and also inadvertently (through posts by vapers) promoting the use of e-cigarettes. Twitter has a "paid" advertising policy prohibiting the promotion of tobacco products, accessories, and branding (including e-cigarettes) [67]. The policy, however, does not relate to individual account holder's content, fan pages, or groups. The boundaries between owned, paid, earned, and shared content have become increasingly more blurred [68], with evidence suggesting influencers are being used to circumvent social media policies $[69,70]$. In the absence of regulations controlling online promotions and formal gateways restricting access to content, posts on social media platforms such as Twitter can reach and potentially influence both e-cigarette users and nonusers alike [51]. Exploring opportunities to further restrict the commercial promotion of these devices (ie, unpaid promotion from commercial accounts) on Twitter and other social media platforms is required, and working with social media platforms to voluntarily employ these restrictions is one possible solution [71].

This study found the proportion of posts specifically promoting e-cigarette products for purchase decreased in 2018 (Multimedia Appendix 1), although this correlates with a relative decline in Twitter use by Australians in comparison to other larger and growing platforms. Due to the increased popularity of Instagram over recent years, and more recently TikTok, it would be valuable to investigate e-cigarette-related promotional content posted to these platforms. Instagram and TikTok are primarily photo and video-sharing social networking services; therefore, these platforms may be more desirable and more highly accessed than Twitter to share this type of content.

A product for therapeutic use, such as smoking cessation or alleviation of nicotine withdrawal, must be registered with the
Therapeutic Goods Administration to be sold lawfully in Australia [2]. At present, no heated tobacco nor nicotine vaporizer has been approved by the Therapeutic Goods Administration and therefore should not be promoted as a smoking cessation product. Continued monitoring of Australian e-cigarette retailers to ensure misleading health and smoking cessation claims are not being made is therefore important so as not to contribute further to the confusion regarding e-cigarette safety and efficacy.

\section{Limitations}

Several limitations need to be considered when interpreting the results of this study. This study reflects data from one social media platform, Twitter, as its data are mostly public and easily accessible to researchers, whereas some other social media platforms are not as readily accessible [72]. However, the TrISMA infrastructure makes Australian-specific historical Twitter data accessible in a way most other social media platforms do not. This is not an indication that other social media platforms are not spaces where e-cigarettes are discussed by Australians, but only that these activities are not always as visible to researchers. The search strategy included several popular terms used to describe e-cigarettes and vaping practices; however, emerging and variations of slang terms may have been overlooked. The investigation focused only on tweets that included an image. Therefore, these results may not be reflective of all tweets by Australian users. Lastly, we relied on TrISMA's programmed bot filtering processes occurring at the level of the user before tweets were collected to remove questionable accounts. Future studies examining Twitter data are encouraged to apply denoising techniques after data collection [73].

\section{Conclusions}

Despite Australia's cautious approach toward e-cigarettes and the limited evidence supporting e-cigarettes as an efficacious smoking cessation aid, it is evident that there is a concerted effort by some Twitter users to promote these devices as a harmless, health-conducive, smoking cessation product. Further, Twitter is being used in an attempt to circumvent Australian regulation and advocate for a liberal approach to personal vaporizers. The borderless nature of social media presents a clear challenge for enforcing Article 13 of the WHO FCTC. Evidence suggests a relationship exists between e-cigarette advertising exposure and uptake, and social media is now being used to generate favorable attitudes towards vaporizer products. As "digital media" consumption has increased, content that was previously inaccessible due to conventional advertising regulations, such as tobacco advertising, is now visible, and traditional tobacco control regulations are no longer adequate. The internet is the perfect platform to promote e-cigarettes and novel nicotine products, even in a highly regulated country such as Australia. Countering the advertising and promotion of these products is a public health challenge that will require cross-border cooperation with other WHO FCTC parties.

\section{Acknowledgments}

This work was supported by a Healthway Exploratory Research Grant (grant number 32803) and an Australian Government Research Training Program Scholarship. The scholarship is provided by the Commonwealth of Australia to support the general 
living costs for students (KM) undertaking Research Doctorate studies. This research was also supported by the Faculty of Health Sciences, Curtin University 2019 Health Sciences Summer Scholarship Initiative. The scholarship is provided to support the general living costs of undergraduate students (KT) to undertake a research project. All funders had no role in the design of the study; in the collection, analyses, or interpretation of data; in the writing of the manuscript; and in the decision to publish the results. This research was also supported by infrastructure provided through the Australian Research Council-funded project TrISMA: Tracking Infrastructure for Social Media Analysis (LIEF grant LE140100148).

We would like to acknowledge Dr Kevin Chai, Dr Alkim Ozaygen, and Dr Yun Zhao from Curtin University for their assistance with data collection and statistical analyses. We would also like to thank the Cancer Council Western Australia, Australian Council on Smoking and Health, Public Health Advocacy Institute of Western Australia, and Royal Australian College of General Practitioners Western Australia for being members of the study's advisory committee; they provided advice to the research team to help guide the implementation of the project, use of generated data, and dissemination of the research findings.

\section{Authors' Contributions}

JJ, BM, TL, KW, and KM acquired the funding. KM, JJ, BM, TL, and KW conceptualized the study and methodology. KM performed project administration, curated the data, and wrote the original draft of the manuscript. JJ, BM, and TL supervised the study. KM and KT performed the formal analysis. Review and editing of the manuscript was performed by BF, JJ, BM, KW, and TL.

\section{Conflicts of Interest}

BF is a member of the NHMRC Electronic Cigarettes Working Committee (May 2020). She has received consulting payment for e-cigarette policy review for the New South Wales National Heart Foundation (December 2019). She had travel expenses (flight and registration) reimbursed to attend Oceania Tobacco Control Conference 2017 to present on e-cigarette and cessation. She provided her opinion (unpaid) at the Australian Parliament's Standing Committee on Health, Aged Care and Sport public hearing into the Use and Marketing of Electronic Cigarettes and Personal Vaporisers (September 8, 2017). She led a contract on e-cigarette regulation in Australia for the Commonwealth Department of Health (2016). She had travel expenses reimbursed by National Taiwan University for presenting on e-cigarette regulation (2016). The other authors have no conflicts to declare.

\section{Multimedia Appendix 1}

Coding framework.

[DOCX File, 20 KB-Multimedia Appendix 1]

\section{References}

1. Legal status of electronic cigarettes in Australia. Quit Victoria. 2016. URL: https://www.quit.org.au/documents/77/ legal-status-electronic-cigarettes-australia.pdf [accessed 2019-05-27]

2. Therapeutic Goods Administration. Electronic cigarettes. Australian Government Department of Health. 2019. URL: https:/ /www.tga.gov.au/community-qa/electronic-cigarettes [accessed 2019-06-24]

3. NicoPharm. 2019. URL: http://nicopharm.com.au/ [accessed 2019-11-06]

4. Greenhalgh EM, Grace C, Scollo MM. 18B.8: Legal status in Australia. Tobacco in Australia: Facts and issues. Melbourne, Victoria, Australia: Cancer Council Victoria; 2019. URL: https://www.tobaccoinaustralia.org.au/chapter-18-harm-reduction/ indepth-18b-e-cigarettes/18b-9-legal-status [accessed 2019-11-06]

5. Australian Border Force. Tobacco. Prohibited goods. 2020. URL: https://www.abf.gov.au/ importing-exporting-and-manufacturing/prohibited-goods/categories/tobacco [accessed 2020-10-16]

6. World Health Organization. Guidelines for implementation of Article 13: Guidelines on tobacco advertising, promotion and sponsorship. WHO Framework Convention on Tobacco Control. 2008. URL: https://www.who.int/fctc/guidelines/ adopted/article 13/en/ [accessed 2020-10-16]

7. NSW Government. Electronic cigarettes. 2019. URL: https://www.health.nsw.gov.au/tobacco/Pages/e-cigarettes.aspx [accessed 2020-03-04]

8. Government of South Australia. Selling e-cigarettes in South Australia - retailer fact sheet. Drug and Alcohol Services South Australia. 2019. URL: https://www.sahealth.sa.gov.au/wps/wcm/connect/f3a6e0fa-762a-4be0-acfa-09a9a11e1b4c/ Selling+E-Cigarettes+in+SA+-+Retailer+Fact+Sheet+200319.pdf [accessed 2020-03-04]

9. Tasmanian Government. Tobacco control - Electronic cigarettes. 2019. URL: https://www.dhhs.tas.gov.au/publichealth/ tobacco_control/electronic-cigarettes [accessed 2020-03-04]

10. Queensland Government. Electronic cigarettes. Tobacco laws in Queensland. 2019. URL: https://www.health.qld.gov.au/ public-health/topics/atod/tobacco-laws/electronic-cigarettes [accessed 2020-03-04]

11. Australian Institute of Health and Welfare. National Drug Strategy Household Survey. Canberra: AIHW; 2020. URL: https:/ /www.aihw.gov.au/about-our-data/our-data-collections/ 
national-drug-strategy-household-survey\#: :text=The\%20National\%20Drug\%20Strategy\%20Household,alcohol\%20and\%20other\%20drug\%20use [accessed 2020-10-16]

12. McCausland K, Maycock B, Jancey J. The messages presented in online electronic cigarette promotions and discussions: a scoping review protocol. BMJ Open 2017;7(11):e018633. [doi: 10.1136/bmjopen-2017-018633] [Medline: 29122804]

13. Chen AT, Zhu S, Conway M. What Online Communities Can Tell Us About Electronic Cigarettes and Hookah Use: A Study Using Text Mining and Visualization Techniques. J Med Internet Res 2015;17(9):e220 [FREE Full text] [doi: 10.2196/jmir.4517] [Medline: 26420469]

14. Ayers JW, Leas EC, Allem J, Benton A, Dredze M, Althouse BM, et al. Why do people use electronic nicotine delivery systems (electronic cigarettes)? A content analysis of Twitter, 2012-2015. PLoS One 2017;12(3):e0170702 [FREE Full text] [doi: 10.1371/journal.pone.0170702] [Medline: 28248987]

15. Allem J, Escobedo P, Chu K, Boley Cruz T, Unger JB. Images of Little Cigars and Cigarillos on Instagram Identified by the Hashtag \#swisher: Thematic Analysis. J Med Internet Res 2017;19(7):e255 [FREE Full text] [doi: 10.2196/jmir.7634] [Medline: 28710057]

16. Hootsuite, We Are Social. Digital 2019 Australia. Datareportal. 2019. URL: https://datareportal.com/reports/ digital-2019-australia?rq=australia [accessed 2019-11-06]

17. Bruns A, Stieglitz S. Quantitative Approaches to Comparing Communication Patterns on Twitter. Journal of Technology in Human Services 2012;30(3-4):160-185. [doi: 10.1080/15228835.2012.744249]

18. Hébert ET, Case KR, Kelder SH, Delk J, Perry CL, Harrell MB. Exposure and Engagement With Tobacco- and E-Cigarette-Related Social Media. J Adolesc Health 2017;61(3):371-377 [FREE Full text] [doi: 10.1016/j.jadohealth.2017.04.003] [Medline: 28669801]

19. McCausland K, Maycock B, Leaver T, Jancey J. The Messages Presented in Electronic Cigarette-Related Social Media Promotions and Discussion: Scoping Review. J Med Internet Res 2019;21(2):e11953 [FREE Full text] [doi: 10.2196/11953] [Medline: $\underline{30720440]}$

20. Kwon M, Park E. Perceptions and Sentiments About Electronic Cigarettes on Social Media Platforms: Systematic Review. JMIR Public Health Surveill 2020;6(1):e13673 [FREE Full text] [doi: 10.2196/13673] [Medline: 31939747]

21. Vogel EA, Ramo DE, Rubinstein ML, Delucchi KL, Darrow S, Costello C, et al. Effects of Social Media on Adolescents' Willingness and Intention to Use E-Cigarettes: An Experimental Investigation. Nicotine Tob Res 2020. [doi: 10.1093/ntr/ntaa003] [Medline: 31912147]

22. Camenga D, Gutierrez KM, Kong G, Cavallo D, Simon P, Krishnan-Sarin S. E-cigarette advertising exposure in e-cigarette naïve adolescents and subsequent e-cigarette use: A longitudinal cohort study. Addict Behav 2018;81:78-83 [FREE Full text] [doi: 10.1016/j.addbeh.2018.02.008] [Medline: 29432916]

23. Pokhrel P, Fagan P, Herzog TA, Laestadius L, Buente W, Kawamoto CT, et al. Social media e-cigarette exposure and e-cigarette expectancies and use among young adults. Addict Behav 2018;78:51-58 [FREE Full text] [doi: 10.1016/j.addbeh.2017.10.017] [Medline: 29127784]

24. Wagoner KG, Reboussin DM, King JL, Orlan E, Cornacchione Ross J, Sutfin EL. Who Is Exposed to E-Cigarette Advertising and Where? Differences between Adolescents, Young Adults and Older Adults. Int J Environ Res Public Health 2019;16(14) [FREE Full text] [doi: 10.3390/ijerph16142533] [Medline: 31315189]

25. Ghosh A, Coakley RC, Mascenik T, Rowell TR, Davis ES, Rogers K, et al. Chronic E-Cigarette Exposure Alters the Human Bronchial Epithelial Proteome. Am J Respir Crit Care Med 2018;198(1):67-76 [FREE Full text] [doi:

10.1164/rccm.201710-20330C] [Medline: 29481290]

26. The Lancet. E-cigarettes: time to realign our approach? Lancet 2019;394(10206):1297. [doi: 10.1016/S0140-6736(19)32277-9] [Medline: $\underline{31609211]}$

27. Muthumalage T, Prinz M, Ansah KO, Gerloff J, Sundar IK, Rahman I. Inflammatory and Oxidative Responses Induced by Exposure to Commonly Used e-Cigarette Flavoring Chemicals and Flavored e-Liquids without Nicotine. Front Physiol 2017;8:1130 [FREE Full text] [doi: 10.3389/fphys.2017.01130] [Medline: 29375399]

28. Bruns A, Burgess J, Banks J, Tjondronegoro D, Dreiling A, Hartley J, et al. TrISMA: Tracking Infrastructure for Social Media Analysis. QUT Digital Media Research Centre. 2016. URL: http://trisma.org/ [accessed 2019-06-01]

29. Chu K, Allem J, Cruz TB, Unger JB. Vaping on Instagram: cloud chasing, hand checks and product placement. Tob Control 2016;26(5):575-578 [FREE Full text] [doi: 10.1136/tobaccocontrol-2016-053052] [Medline: 27660111]

30. Laestadius LI, Wahl MM, Cho YI. \#Vapelife: An Exploratory Study of Electronic Cigarette Use and Promotion on Instagram. Subst Use Misuse 2016;51(12):1669-1673. [doi: 10.1080/10826084.2016.1188958] [Medline: 27484191]

31. Laestadius LI, Wahl MM, Pokhrel P, Cho YI. From Apple to Werewolf: A content analysis of marketing for e-liquids on Instagram. Addict Behav 2019;91:119-127 [FRE Full text] [doi: 10.1016/j.addbeh.2018.09.008] [Medline: $\underline{30253933}$ ]

32. Lee A, Hart J, Sears C, Walker K, Siu A, Smith C. A picture is worth a thousand words: Electronic cigarette content on Instagram and Pinterest. Tob Prev Cessat 2017;3 [FREE Full text] [doi: 10.18332/tpc/74709] [Medline: 28815224]

33. Cortese DK, Szczypka G, Emery S, Wang S, Hair E, Vallone D. Smoking Selfies: Using Instagram to Explore Young Women's Smoking Behaviors. Social Media + Society 2018;4(3):1-8 [FREE Full text] [doi: 10.1177/2056305118790762]

34. Ritter SL. Heating up the debate: e-cigarettes and Instagram. Electronic Thesis and Dissertation Repository. 2015. URL: https://ir.lib.uwo.ca/etd/3350 [accessed 2019-06-01] 
35. Cole-Lewis H, Pugatch J, Sanders A, Varghese A, Posada S, Yun C, et al. Social Listening: A Content Analysis of E-Cigarette Discussions on Twitter. J Med Internet Res 2015;17(10):e243 [FREE Full text] [doi: 10.2196/jmir.4969] [Medline: 26508089]

36. Unger JB, Escobedo P, Allem J, Soto DW, Chu K, Cruz T. Perceptions of Secondhand E-Cigarette Aerosol Among Twitter Users. Tob Regul Sci 2016;2(2):146-152 [FREE Full text] [doi: 10.18001/TRS.2.2.5] [Medline: 28090560]

37. RANDOM.ORG. Random sequence generator. 2020. URL: https://www.random.org/sequences/ [accessed 2019-09-10]

38. McBride DM, Anne Dosher B. A comparison of conscious and automatic memory processes for picture and word stimuli: a process dissociation analysis. Conscious Cogn 2002;11(3):423-460. [doi: 10.1016/s1053-8100(02)00007-7] [Medline: 12435377]

39. Chang Y, Yu H, Lu H. Persuasive messages, popularity cohesion, and message diffusion in social media marketing. Journal of Business Research 2015;68(4):777-782. [doi: 10.1016/j.jbusres.2014.11.027]

40. Townsend L, Wallace C. Social media research: A guide to ethics. The University of Aberdeen. 2016. URL: https://www. gla.ac.uk/media/Media 487729 smxx.pdf [accessed 2020-10-16]

41. Schreier M. Qualitative content analysis in practice. London, England: Sage Publications, Inc; 2012.

42. Highfield T, Leaver T. A methodology for mapping Instagram hashtags. First Monday 2015;20(1) [FREE Full text] [doi: 10.5210/fm.v20i1.5563]

43. Krippendorff K. Content analysis: An introduction to its methodology. Thousand Oaks, CA: Sage Publications, Inc; 2004.

44. Collins L, Glasser AM, Abudayyeh H, Pearson JL, Villanti AC. E-Cigarette Marketing and Communication: How E-Cigarette Companies Market E-Cigarettes and the Public Engages with E-cigarette Information. Nicotine Tob Res 2019;21(1):14-24 [FREE Full text] [doi: 10.1093/ntr/ntx284] [Medline: 29315420]

45. Paek H, Reid LN, Jeong HJ, Choi H, Krugman D. Five decades of promotion techniques in cigarette advertising: a longitudinal content analysis. Health Mark Q 2012;29(1):1-17. [doi: 10.1080/07359683.2011.623087] [Medline: 22416922]

46. E-cigarette market - Growth, trends and forecast (2020-2025). Mordor Intelligence. 2020. URL: https://www. mordorintelligence.com/industry-reports/global-e-cigarettes-market-industry [accessed 2020-09-15]

47. Mackey TK, Miner A, Cuomo RE. Exploring the e-cigarette e-commerce marketplace: Identifying Internet e-cigarette marketing characteristics and regulatory gaps. Drug Alcohol Depend 2015;156:97-103. [doi: 10.1016/j.drugalcdep.2015.08.032] [Medline: 26431794]

48. Gurram N, Thomson G, Wilson N, Hoek J. Electronic cigarette online marketing by New Zealand vendors. N Z Med J 2019;132(1506):20-33. [Medline: 31778369]

49. Knopf A. FDA warns against underage use of JUUL, cracks down on sales. The Brown University Child and Adolescent Behavior Letter 2018;34(6):3-3. [doi: 10.1002/cbl.30300]

50. Soule EK, Sakuma KK, Palafox S, Pokhrel P, Herzog TA, Thompson N, et al. Content analysis of internet marketing strategies used to promote flavored electronic cigarettes. Addict Behav 2019;91:128-135. [doi: 10.1016/j.addbeh.2018.11.012] [Medline: $\underline{30606627]}$

51. Allem J, Majmundar A, Dharmapuri L, Cruz TB, Unger JB. E-liquid-related posts to Twitter in 2018: Thematic analysis. Addict Behav Rep 2019;10:100196 [FREE Full text] [doi: 10.1016/j.abrep.2019.100196] [Medline: 31431917]

52. Farsalinos K, Romagna G, Tsiapras D, Kyrzopoulos S, Spyrou A, Voudris V. Impact of flavour variability on electronic cigarette use experience: an internet survey. Int J Environ Res Public Health 2013;10(12):7272-7282 [FREE Full text] [doi: 10.3390/ijerph10127272] [Medline: 24351746]

53. Chu K, Unger JB, Cruz TB, Soto DW. Electronic Cigarettes on Twitter - Spreading the Appeal of Flavors. Tob Regul Sci 2015;1(1):36-41 [FREE Full text] [doi: 10.18001/TRS.1.1.4] [Medline: 27853734]

54. Hoffman AC, Salgado RV, Dresler C, Faller RW, Bartlett C. Flavour preferences in youth versus adults: a review. Tob Control 2016;25(Suppl 2):ii32-ii39 [FREE Full text] [doi: 10.1136/tobaccocontrol-2016-053192] [Medline: 27633764]

55. Tsai J, Walton K, Coleman BN, Sharapova SR, Johnson SE, Kennedy SM, et al. Reasons for Electronic Cigarette Use Among Middle and High School Students - National Youth Tobacco Survey, United States, 2016. MMWR Morb Mortal Wkly Rep 2018;67(6):196-200 [FREE Full text] [doi: 10.15585/mmwr.mm6706a5] [Medline: 29447148]

56. Pepper JK, Ribisl KM, Brewer NT. Adolescents' interest in trying flavoured e-cigarettes. Tob Control 2016;25(Suppl 2):ii62-ii66 [FREE Full text] [doi: 10.1136/tobaccocontrol-2016-053174] [Medline: 27633762]

57. Soule EK, Rosas SR, Nasim A. Reasons for electronic cigarette use beyond cigarette smoking cessation: A concept mapping approach. Addict Behav 2016;56:41-50 [FREE Full text] [doi: 10.1016/j.addbeh.2016.01.008] [Medline: 26803400]

58. Farsalinos KE, Romagna G, Tsiapras D, Kyrzopoulos S, Voudris V. Characteristics, perceived side effects and benefits of electronic cigarette use: a worldwide survey of more than 19,000 consumers. Int J Environ Res Public Health 2014;11(4):4356-4373 [FREE Full text] [doi: 10.3390/ijerph110404356] [Medline: 24758891]

59. Tomashefski A. The perceived effects of electronic cigarettes on health by adult users: A state of the science systematic literature review. J Am Assoc Nurse Pract 2016;28(9):510-515. [doi: 10.1002/2327-6924.12358] [Medline: 26997487]

60. Czoli CD, Fong GT, Mays D, Hammond D. How do consumers perceive differences in risk across nicotine products? A review of relative risk perceptions across smokeless tobacco, e-cigarettes, nicotine replacement therapy and combustible cigarettes. Tob Control 2017;26(e1):e49-e58. [doi: 10.1136/tobaccocontrol-2016-053060] [Medline: 27625408] 
61. Gorukanti A, Delucchi K, Ling P, Fisher-Travis R, Halpern-Felsher B. Adolescents' attitudes towards e-cigarette ingredients, safety, addictive properties, social norms, and regulation. Prev Med 2017;94:65-71 [FREE Full text] [doi: 10.1016/j.ypmed.2016.10.019] [Medline: 27773711]

62. Strong DR, Messer K, White M, Shi Y, Noble M, Portnoy DB, et al. Youth perception of harm and addictiveness of tobacco products: Findings from the Population Assessment of Tobacco and Health Study (Wave 1). Addict Behav 2019;92:128-135 [FREE Full text] [doi: 10.1016/j.addbeh.2018.12.005] [Medline: 30623806]

63. Allem J, Ferrara E, Uppu SP, Cruz TB, Unger JB. E-Cigarette Surveillance With Social Media Data: Social Bots, Emerging Topics, and Trends. JMIR Public Health Surveill 2017;3(4):e98 [FREE Full text] [doi: 10.2196/publichealth.8641] [Medline: 29263018]

64. Farrimond H. A typology of vaping: Identifying differing beliefs, motivations for use, identity and political interest amongst e-cigarette users. Int J Drug Policy 2017;48:81-90. [doi: 10.1016/j.drugpo.2017.07.011] [Medline: 28810158]

65. McCausland K, Jancey J, Leaver T, Wolf K, Freeman B, Maycock B. Motivations for use, identity and the vaper subculture: a qualitative study of the experiences of Western Australian vapers. BMC Public Health 2020 Oct 15;20(1):1552 [FREE Full text] [doi: 10.1186/s12889-020-09651-z] [Medline: $\underline{33059652]}$

66. Chang H. A new perspective on Twitter hashtag use: Diffusion of innovation theory. Proceedings of the American Society for Information Science and Technology 2011;47(1):1-4. [doi: 10.1002/meet.14504701295]

67. Twitter. Tobacco and tobacco accessories. Prohibited content policies. 2019. URL: https://business.twitter.com/en/help/ ads-policies/prohibited-content-policies/tobacco-and-tobacco-accessories.html [accessed 2020-03-04]

68. Macnamara J, Lwin M, Adi A, Zerfass A. 'PESO' media strategy shifts to 'SOEP': Opportunities and ethical dilemmas. Public Relations Review 2016;42(3):377-385. [doi: 10.1016/j.pubrev.2016.03.001]

69. Vassey J, Metayer C, Kennedy CJ, Whitehead TP. \#Vape: Measuring E-Cigarette Influence on Instagram With Deep Learning and Text Analysis. Front. Commun 2020;4(75). [doi: 10.3389/fcomm.2019.00075]

70. Kirkham C. Exclusive: Philip Morris suspends social media campaign after Reuters exposes young 'influencers'. Reuters. 2019. URL: https://www.reuters.com/article/us-philipmorris-ecigs-instagram-exclusiv/ exclusive-philip-morris-suspends-social-media-campaign-after-reuters-exposes-young-influencers-idUSKCN1SH02K [accessed 2020-03-04]

71. Freeman B. New media and tobacco control. Tob Control 2012;21(2):139-144. [doi: 10.1136/tobaccocontrol-2011-050193] [Medline: 22345236]

72. Weller K, Bruns A, Burgess J, Mahrt M, Puschmann C. Twitter and society. New York, NY: Peter Lang Publishing, Inc; 2014. URL: http://snurb.info/files/2014/

Twitter\%20and\%20Society\%20-\%20Metrics\%20for\%20Understanding\%20Communication\%20on\%20Twitter\%20\%282014\%29. pdf [accessed 2019-06-24]

73. Allem J, Ferrara E. The Importance of Debiasing Social Media Data to Better Understand E-Cigarette-Related Attitudes and Behaviors. J Med Internet Res 2016;18(8):e219 [FREE Full text] [doi: 10.2196/jmir.6185] [Medline: 27507563]
Abbreviations
e-cigarette: electronic cigarette
GIF: graphic interchange format
TrISMA: Tracking Infrastructure for Social Media Analysis
WHO FCTC: World Health Organization Framework Convention on Tobacco Control

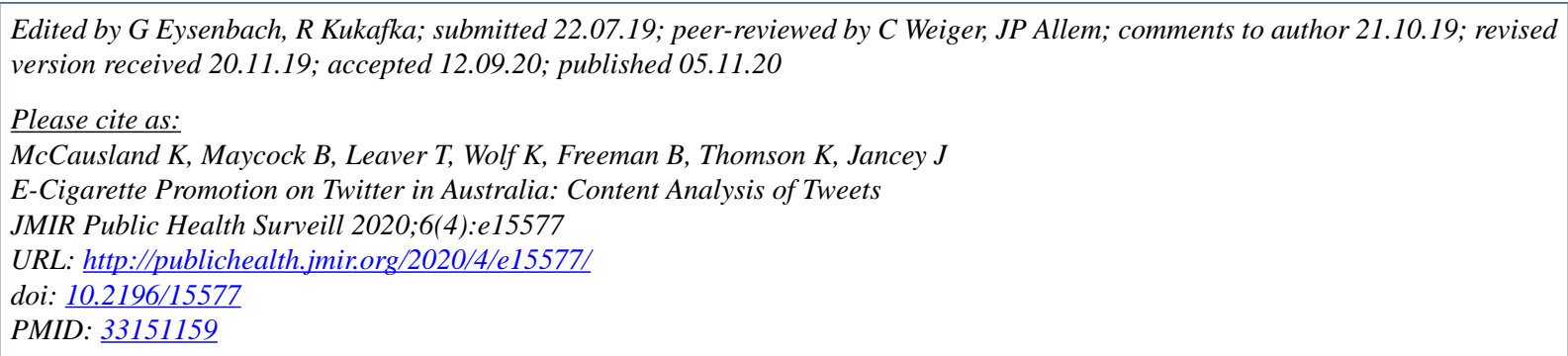

(CKahlia McCausland, Bruce Maycock, Tama Leaver, Katharina Wolf, Becky Freeman, Katie Thomson, Jonine Jancey. Originally published in JMIR Public Health and Surveillance (http://publichealth.jmir.org), 05.11.2020. This is an open-access article distributed under the terms of the Creative Commons Attribution License (https://creativecommons.org/licenses/by/4.0/), which permits unrestricted use, distribution, and reproduction in any medium, provided the original work, first published in JMIR Public 
Health and Surveillance, is properly cited. The complete bibliographic information, a link to the original publication on http://publichealth.jmir.org, as well as this copyright and license information must be included. 